\title{
Israeli Mental Health Professionals' Attitudes Towards Dissociative Disorders, Reported Incidence and Alternative Diagnoses Considered
}

\author{
Eli Somer, PhD
}

\begin{abstract}
Clinical diagnoses of dissociative disorders (DDs), including Dissociative Identity Disorder (DID), are controversial because there are mental health professionals in North America and elsewhere who are skeptical about whether these psychiatric disorders actually exist. This paper explores the attitudes of mental health professionals in Israel toward DDs and DID through a survey of 211 practicing clinicians (return rate of $39.5 \%$ ). Of the sample, $95.5 \%$ scored at or above the point on a 5-point Likert scale measuring belief in the validity of DDs $(\mathrm{m}=4.17, \mathrm{SD}=0.78) ; 84.5 \%$ declared at least a moderate belief in the validity of DID $(\mathrm{M}=3.5, \mathrm{~S} . \mathrm{D} .=0.97)$. The average Israeli clinician surveyed had made 4.8 career-long DD diagnoses $($ S.D. $=18.06)$ and carried an average of 1.05 DD patients in his/her caseload (S.D. = 2.86). DID had a career-long diagnosis frequency of 0.14 patients per clinician (S.D. $=0.59)$ and was currently seen at a frequency of 0.03 cases per clinician $($ S.D. $=0.20)$. The five most frequently considered alternative diagnoses to DID in Israel were Borderline Personality Disorder (24\%), Psychotic Disorder/Schizophrenia (23\%), PTSD/Anxiety Disorder (10\%), Malingering (8\%) and Depressive Disorder (7\%). The findings suggest that attitudes of Israeli clinicians are similar to those of North American clinicians despite the geographical and cultural differences between them. [Article copies available for a fee from The Haworth Document Delivery Service: 1-800-342-9678. E-mail address: <getinfo@haworthpressinc.com> Website: <http://www.HaworthPress.com>]
\end{abstract}

Eli Somer is Associate Professor of Psychology, the Faculty of Social Welfare and Health Studies, University of Haifa, Mt. Carmel, Haifa 31905, Israel, and Maytal-Israel Institute for Treatment and Study of Stress.

Address correspondence to: somer@research.haifa.ac.il or to School of Social Work, University of Haifa, Mt. Carmel, Haifa 31905, Israel.

The author wishes to thank Hagit Wiesel for her help in conducting this study.

Journal of Trauma \& Dissociation, Vol. 1(1) 2000

(c) 2000 by The Haworth Press, Inc. All rights reserved. 
KEYWORDS. Dissociative disorders, dissociation, dissociative identity disorder, attitudes, beliefs, skepticism, survey, professionals, psychologists, psychiatrists, Israel

For more than a decade, the topic of dissociative disorders has been the focus of a heated debate among mental health professionals. The debate has accompanied a continuously increasing number of cases of dissociative disorders (DDs) and their more extreme manifestation, Dissociative Identity Disorder (DID) (Fahy, 1988; Putnam, 1989, 1996). Hayes and Mitchell (1994) showed that skepticism about DDs could be directly related to inaccuracy in diagnosing DID, which can promote false negative errors. The authors also found that skepticism and knowledge about DID were inversely related. This finding means that some DD patients may not be receiving efficacious treatments that follow acceptable clinical guidelines. The controversy centers on whether DDs, and particularly DID are valid clinical diagnoses (Mersky, 1992; Spanos, 1996) and on the meaning of the increased number of diagnosed cases (Horevitz, 1994). Some writers in the field have claimed that clinicians are so fascinated with the dissociation model that they inadvertently elicit these clinical phenomena during therapy (Bowers, 1991; Frankel, 1990). Others have suggested that the dramatic increase in the diagnosis of DID reflects a North American professional fashion trend that had developed into a form of social hysteria (Aldridge-Morris, 1989; Radwin, 1991). They maintain that dissociative multiplicity is socially constructed because it is "a context-bound goal-directed social behavior geared to the expectations of significant others" (Spanos, 1994). DID patients have also been seen as displaying symptoms of "hysterical acting out for secondary gain" (Thigpen \& Cleckley, 1984, p. 63).

Trauma clinicians, who claim that the increasing number of reported cases of DD is explained by the improved psychodiagnostic skills of clinicians, have remained on the defensive. Mental health professionals are better informed now about the mental fragmentation process that victims of child abuse defensively employ and about the complex psychopathology that results (Putnam, 1989; Braun \& Sachs, 1985).

The recent development of valid and reliable screening instruments has enabled the accumulation of data supporting the reliability and validity of the diagnosis of DID and other DDs (Carlson \& Putnam, 
1993; Steinberg, 1993). The Dissociative Experiences Scale (DES), first developed in English in 1986, is now available in 18 languages, indicating the extent of the international interest in this clinical phenomenon (Bowman, 1996).

Despite the increase in knowledge and the international familiarity with DD, controversy, particularly over the existence of DID as a clinical and diagnostic entity, has continued. A survey of 62 therapists who have treated patients with DID in the United States found that $98 \%$ of respondents reported that they had encountered skepticism from fellow professionals. Of these therapists, $78 \%$ had encountered intense skepticism, deliberate interference with the clinical care of patients and acts of harassment against the patient and/or therapist (Dell, 1988). Commenting on that paper, Bliss (1988) added that he ran a mini-survey among his colleagues and inquired about professional hostility to the notion that many patients had multiple personalities. He concluded that colleagues thought that skepticism is warranted until the documentation is conclusive. He also noted that DID is not acceptable to any of the many schools within psychiatry, that many experienced psychiatrists claim they have never seen a case of DID, that these patients are theatrical if not deceptive, and that gullible professionals have gotten caught up in the construction of this psychological phenomenon.

Takahashi (1990) reported no cases of DID among 489 Japanese psychiatric inpatients [diagnosed using the Diagnostic and Statistical Manual of Mental Disorders (DSM-III) and the DSM-III Revised (DSM-III-R)], although seven subjects classified as schizophrenics claimed a change of identity. Commentaries on Takahashi's paper noted problems within the report including lack of a valid diagnostic instrument, the short hospital stays of the patients, and other deficiencies in the areas of confirmation bias and interrater reliability (Frischolz \& Braun, 1990; Martinez-Taboas, 1990; Ross, 1990; Van der Hart, 1990). A report from New Zealand indicated that many qualified mental health professionals at three universities believe DID does not exist or is extremely rare, yet two years later the author contacted local therapists who dealt with victims of abuse and quickly located a number of DID cases (Altrocchi, 1992).

In a letter to the editor of the British Journal of Psychiatry, Mersky (1993) reported that among 38 Canadian colleagues, who were admittedly sympathetic to his views, 33 either believed DID was over-diag- 
nosed or did not believe in it at all. Based on changes in the reported attitudes of people who had attended one of his lectures, he further concluded that "informed" individuals were likely either to reject or restrict the diagnosis of DID.

In their letter to the editor of Hospital and Community Psychiatry, Clancy, Yates, and Cadoret (1993) reported initial results from an attitude survey suggesting that U.S. mental health professionals' opinions about DID were affected by college training, theory of practice, and clinical experience. A methodologically sound national survey of 207 American mental health professionals supported the hypothesis that skepticism and knowledge about DID are inversely related, and that skepticism is directly related to inaccuracy in diagnosing DID (Hayes \& Mitchell, 1994). Of their sample, 24\% scored at or above the theoretical midpoint of their skepticism scale, and $13 \%$ were outright skeptical about the validity of DID. An analysis of 1120 survey forms returned by American VA psychologists and psychiatrists revealed that more than $97 \%$ of respondents believed in DDs, while $80 \%$ reported a belief in DID (Dunn et al., 1994). The results suggested a tendency for younger staff with less professional experience to indicate a higher frequency of belief in DID. Psychiatrists in that sample demonstrated less certainty about the existence of DID than did psychologists. Out of 180 Canadian psychiatrists, $28 \%$ doubted the existence of DID. A mean of $42.2 \%$ of respondents had never seen a patient with DID. On average, 3.78 DID patients had been seen by Canadian psychiatrists, but the total mean number of cases newly diagnosed per psychiatrist was only 1.21 (with a relatively high variability) (Mai, 1995). Data from 425 respondents from a random-selection sample obtained from the American Psychological Association revealed that only $8 \%$ believed that DID does not exist. The majority of respondents (59\%) thought that DID is rare and occurs at rates between $0.1 \%$ and $0.5 \%$ in the general population. Many respondents (65\%) believed that DID occurs cross-culturally. Approximately one half of all respondents believed that they had encountered a client with DID (Cormier \& Thelen, 1998). Recently, Pope et al. (1999) reported about the attitudes of 301 randomly sampled board-certified American psychiatrists toward DSM-IV categories of dissociative amnesia and dissociative identity disorder. About a third of respondents replied that these diagnoses should be included without reservation in DSM-IV; the modal response was that they should be included only as proposed 
diagnoses. About one quarter of respondents felt that diagnoses of these two DDs were supported by strong evidence of scientific validity. The authors concluded they found little consensus regarding the diagnostic status or scientific validity of dissociative amnesia and dissociative identity disorder.

Skepticism regarding diagnosis and treatment of DD/DID was reported to be rampant outside North America and the Netherlands (van der Hart, 1993). Van der Hart's paper was particularly critical of British mainstream psychiatry. He described the atmosphere in the United Kingdom as unsupportive and specifically named the British Journal of Psychiatry as being exceptionally conservative and as tending to publish positive remarks regarding DID only in the "Letters to the Editor" section. Bowman (1996) identified the British Isles as an "island of stiff resistance to the concept of DID." She claimed that some British psychiatrists would not treat a patient with DD unless the patient accepted a different diagnosis.

A recent survey conducted in Britain sought to test the prevailing view in the United Kingdom academic press that DID either did not exist or was fashionably over-diagnosed by gullible practitioners, influenced by ill-advised North American colleagues. The survey was designed to examine British psychologists' and psychiatrists' attitudes towards the identification and treatment of dissociative disorders (McIntee, 1998). Dissociative disorders had been encountered by $66 \%$ of respondents, of whom $14 \%$ attributed dissociation to iatrogenesis. The 965 British mental health professionals responding to the survey reported having seen a total of 3225 clients with DDs, 526 clients diagnosed as DID, and 596 clients with Dissociative Disorder-Not Otherwise Specified. The estimated life prevalence rates for a British research sample reported a year earlier were $15.2 \%$ for DDs in general and $5.7 \%$ for DID specifically, with clinical profiles resembling those described in the North American literature (Davis \& Davis, 1997).

\section{DATA ON PROFESSIONAL ATTITUDES AND DDS EPIDEMIOLOGY: A GLOBAL REVIEW}

During the past decade studies of the general population of five countries have found DID in $0.5-3 \%$ of the population (Akyuz et al., 1996; Ross, 1991; Vanderlinden et al., 1991; Vanderlinden et al., 1993). Studies conducted in four countries found DID in 3-6\% of 
outpatients (Davis \& Davis, 1995; Graves, 1989; Ross, 1991; ar et al., 1996). Reports from six different countries indicated that rates of DID in psychiatric inpatient populations ranged from $0-12 \%$ with a significant number of findings clustering around 5\%. The rates of all DD among inpatients in international samples ranged from $4 \%$ to $10 \%$ (Knudsen et al., 1995; Latz et al., 1995; Modestin, 1996; Ross, 1991; Takahashi, 1990; Tutkun et al., 1996).

However, the jury is still out concerning the global prevalence of the phenomenon. Skeptics such as Spanos (1996) and Piper (1997) continued to insist that DID strongly appeared to be a culture-bound syndrome, restricted mostly to North America, influenced by the local media and produced by mutual shaping between over-zealous therapists and clients eager to elicit their highly esteemed approval.

Clinical studies that explore epidemiological data and attitude surveys among mental health professionals on dissociative disorders are extremely scarce in non-English-speaking countries. In addition to the Netherlands and Flanders, where the greatest interest in DDs outside of the USA has been noted (Bowman, 1996; van der Hart, 1993), published research on DDs has also been conducted in Puerto Rico and in Turkey. Despite little local awareness of DID and recovered memory, studies from Puerto Rico confirm the clinical constants in the field: the stability of core clinical features across cultures, the association of child abuse and DID, and the corroborative nature of child abuse in these cases (Martinez-Taboas, 1991a, 1991b, 1995a, 1995b).

The research coming from Turkey demonstrated an awareness of DDs that was not preceded by extensive cultural discussion of child abuse and in the absence of North American information (Akyuz et al., 1996; ar, Yargiç, \& Tutkun, 1996).

\section{THE FIELD OF DISSOCIATIVE DISORDERS IN ISRAEL}

Until recently, skepticism about the validity of DD/DID in Israel was widespread. Prior to 1996 no official study program on dissociation was offered in any of Israel's universities or schools of psychotherapy. Since then, the author of this paper has been addressing the topic in a graduate course on trauma at the University of Haifa School of Social Work.

Interest in dissociation in Israel began to emerge in 1987. The 
Dutch dissociation scholar Onno van der Hart gave a two-day workshop on diagnosis and treatment of DID for members of the Israel Hypnosis Society. At the Third National Conference of the same society, the first Hebrew-language scholarly paper on diagnosis and treatment principles of DID was presented (Somer, 1987). Since then a number of clinical and theoretical papers on dissociation have been published in the Hebrew-language professional literature (e.g., Margalit \& Wiztum, 1997a, 1997b; Somer, 1993, 1994, 1995; Somer \& Somer, 1997), and several workshops on the subject have been held during local mental health conferences. In the past several years, approximately 700 people are estimated to have attended educational meetings where DDs were the primary topic. Education on DD rarely attracts much interest when advertised as such. However, interest in child abuse and its aftermath has increased considerably in Israel. Therefore, education on DD is accomplished by placing it in the context of meetings on the sequelae of child abuse.

Two university-based meetings on recovered memories of abuse were held in Israel to date. In June of 1997, a high profile conference was held at Bar Ilan University and featured keynote addresses by dissociation-skeptic and memory scholar Elizabeth Loftus and by Richard Kluft, a leading dissociation scientist. In May of 1999, the University of Haifa held a staged trial on the validity of recovered memories of incest. Recovered memories were discussed by BenTzion Cohen, Head of the University of Haifa School of Social Work, as potentially confabulated material that is very difficult to corroborate. The topic was presented by this author as a product of multiple factors (including dissociation) that inhibit the timely reporting of incest. The dissociation field in Israel, pioneered by a few and ignored by the mainstream, is gradually gaining some recognition.

Despite its general Western orientation, Israel is culturally heterogeneous. It has a large Arab minority, half of its Jewish citizens are immigrants from the Muslim orbit or their descendents, and about $10 \%$ of its Jewish population are recent immigrants from the former Soviet Union. This cultural diversity renders Israel a worthy target for the investigation of a clinical nosology that is suspected by some scholars to be a specific cultural idiom.

The aim of the study reported here was to ascertain the beliefs of Israeli mental health professionals with regard to the validity of DD and the incidence of DD in their caseloads, and also to ascertain the 
common alternative diagnoses considered by Israeli clinicians when encountering a suspected DD/DID patient.

\section{METHOD}

\section{Participants and Procedure}

The directors of seven major mental health services in central and northern Israel were contacted: A psychiatric hospital, a children's hospital, a psychiatric division of a large medical center, the mental health department of the army's medical corps, a large out-patient clinic for children and adolescents, and two rural mental health centers. The sampled agencies are among the leading Israeli mental health institutions. All had gained accreditation by the Ministry of Health as clinical psychology internship sites. The directors of these centers were chosen because of the author's prior personal acquaintance with them. They all were mainstream professionals not known to hold any particular stance on the DD/DID controversy. Their approval and assistance in the administration of the survey was solicited. All seven agreed to cooperate. Two requested that the questionnaires be sent to them and volunteered to distribute the materials and subsequently mail the responses back to the investigator. Five directors preferred that the investigator come to one of their staff meetings to distribute the survey himself. In total, 534 questionnaires were distributed and 211 were completed and returned, which represents a return rate of $39.51 \%$. The sample consisted of 126 women (59.72\%) and 85 men (40.28\%). For the total sample, means for age and years employed as a mental health professional were 43.95 years $($ S.D. $=8.14)$ and $11.68($ S.D. $=7.93)$ respectively. Of those who identified their professions, $106(52 \%)$ were clinical psychologists, $63(30.9 \%)$ were clinical social workers (psychotherapists), 22 (10.8\%) were psychiatrists and $13(6.4 \%)$ were from other mental health professions. This distribution of professions constitutes a representative sample of the psychotherapy providers' community in Israel. Of the respondents, $115(58.4 \%)$ indicated that they worked in an adult out-patient setting, and $29(14.7 \%)$ in an adult in-patient setting. Thirty-four respondents $(17.3 \%)$ worked with children in an out-patient clinic and $19(9.6 \%)$ worked with children in a hospital setting. One-hundred-forty-five respondents $(68.7 \%)$ were 
either born in Israel or were Sephardic Jews of immediate MiddleEastern or North African descent, 42 (19.9\%) were born in Eastern Europe (mostly in the former Soviet Union), 19 (9\%) were born in Western Europe and in English speaking countries, and 5 (2.4\%) were Israeli Arabs. This ethnically diverse cohort of respondents is representative of the ethnic distribution expected among mental health professionals in Israel.

\section{INSTRUMENT}

Participants were presented with criteria for Dissociative Amnesia, Dissociative Fugue, Depersonalization Disorder, Dissociative Identity Disorder (DID) and Dissociative Disorder-Not Otherwise Specified (DDNOS), as defined within the Diagnostic and Statistical Manual of Mental Disorders (4th ed., American Psychiatric Association, 1994). This information was provided to help reduce bias due to differential familiarity with diagnostic criteria. Respondents then answered 10 questions that reflected their belief about the validity of DDs in general, and DID in particular (these items were rated along a 5-point Likert scale). They were asked to estimate their career-long number of newly diagnosed persons with any of the DDs listed, and their current caseload of patients with any of the listed DDs. Respondents were also asked about alternative diagnoses considered when they were uncertain about a DD diagnosis, their sources of knowledge on DDs, and their interest in continued education in the field.

\section{RESULTS}

Of the sample, $95.5 \%(\mathrm{~N}=191)$ scored at or above the theoretical midpoint on a 5-point Likert scale measuring belief in the validity of DDs $(M=4.17$, S.D. $=0.78)$. Figure 1 shows that respondents did not attribute identical validity to DID, and were more divided on their beliefs about this diagnostic classification.

A declaration of at least a moderate belief in the validity of DID was made by $84.5 \%$ of the respondents $(\mathrm{N}=169)(\mathrm{M}=3.5$, S.D. $=0.97)$. A Wilcoxon Sign Rank Test revealed that these means were significantly different (Sign Rank $=2300.5, \mathrm{p}<.0001)$. There was no difference 
FIGURE 1. Degrees of belief in the validities of DDs and DID among Israeli mental health professionals

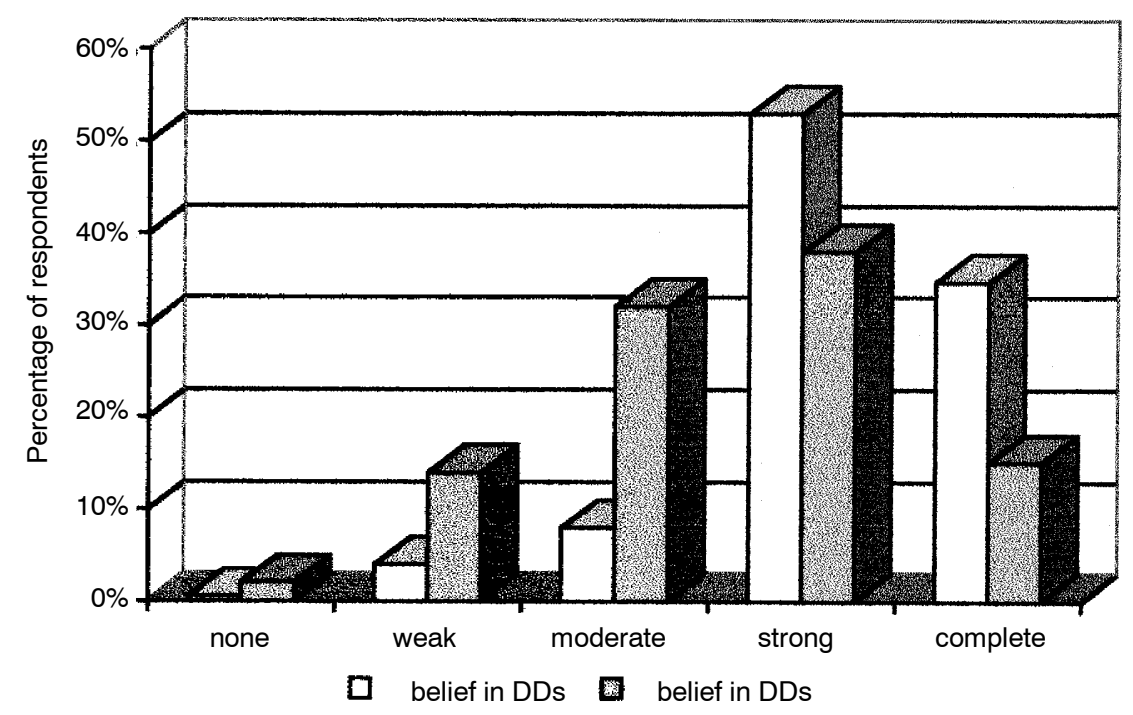

between the various clinical professions as far as the validity assigned to DDs or DID.

No diagnosis or treatment of a DD was reported by 132 clinicians (62.6\% of the total sample). Of these, $55.3 \%$ knew another therapist who had treated an adult DD, and $23.1 \%$ knew a therapist who had worked with a child suffering from DD.

This sample of Israeli clinicians claimed to have made a career-long sum total of 996 diagnoses of DDs $(M=4.8$, S.D. $=18.06)$ and carried a current caseload of 217 patients with DDs $(M=1.05$, S.D. $=2.86)$. Further data on the distribution of diagnosed and (currently) treated DDs across the various clinical professions is shown in Table 1.

No statistical differences between the various clinical professions or ethnic backgrounds of the respondents were found when comparing the mean numbers of diagnosed DDs. Regarding current treatment of DDs, however, clinical psychologists saw more than the expected number of patients with Depersonalization Disorder (Kruksal-Wallis Test Chi square $=8.29, \mathrm{DF}=3, \mathrm{p}=.04$ ).

At least one patient with Dissociative Amnesia had been diagnosed by $42 \%$ of the sample. This disorder was also the most prevalent DD 
TABLE 1. Mean numbers of DD cases estimated to have been diagnosed by a sample of Israeli clinicians and of those currently being treated by them

\begin{tabular}{|c|c|c|c|c|c|c|c|c|}
\hline \multirow[t]{2}{*}{ DD and profession } & \multirow[b]{2}{*}{$\begin{array}{l}\mathrm{N}(\%) \\
\text { clinicians }\end{array}$} & \multicolumn{3}{|c|}{ ever diagnosed } & \multirow[b]{2}{*}{$\begin{array}{l}\mathrm{N}(\%) \\
\text { clinicians }\end{array}$} & \multicolumn{3}{|c|}{ currently treated } \\
\hline & & $\begin{array}{l}\mathrm{N} \\
\text { cases }\end{array}$ & $M$ & SD & & $\begin{array}{l}\mathrm{N} \\
\text { cases }\end{array}$ & M & SD \\
\hline \multicolumn{9}{|l|}{ Dissociative } \\
\hline $\begin{array}{l}\text { Clin. Psych. } \\
(N=106)\end{array}$ & 47 (44\%) & 214 & 4.55 & 6.07 & $25(24 \%)$ & 48 & 1.92 & 1.96 \\
\hline Soc. W. $(N=63)$ & $23(37 \%)$ & 83 & 3.60 & 4.71 & $14(22 \%)$ & 28 & 2.00 & 1.80 \\
\hline Psychiat. ( $\mathrm{N}=22)$ & $10(45 \%)$ & 128 & 12.80 & 30.58 & 3 (14\%) & 11 & 3.67 & 4.62 \\
\hline Other $(\mathrm{N}=12)$ & $6(50 \%)$ & 28 & 4.67 & 7.53 & $1(8 \%)$ & 101 & 10.00 & - \\
\hline Total & $86(42 \%)$ & 453 & 5.77 & 11.52 & $43(21 \%)$ & 97 & 2.26 & 2.42 \\
\hline \multicolumn{9}{|l|}{ Dissociative } \\
\hline Clin. Psych. & $6(6 \%)$ & 6 & 1.00 & 0.63 & 0 & - & - & - \\
\hline Soc. W. & $3(5 \%)$ & 13 & 4.33 & 4.93 & 0 & 0 & - & - \\
\hline Psychiat. & $4(18 \%)$ & 5 & 1.25 & 0.50 & $1(5 \%)$ & 1 & 1.00 & - \\
\hline Other & $1(8 \%)$ & 1 & 1.00 & - & 0 & - & - & - \\
\hline Total & $14(7 \%)$ & 25 & 1.79 & 2.42 & $1(0.5 \%)$ & 1 & 1.00 & - \\
\hline \multicolumn{9}{|l|}{ Depersonalization } \\
\hline Clin. Psych. & 34 (32\%) & 138 & 4.06 & 5.06 & $17(6 \%)$ & 51 & 3.00 & 5.02 \\
\hline Soc. W. & 17 (27\%) & 59 & 3.47 & 5.27 & 9 (14\%) & 9 & 1.00 & - \\
\hline Psychiat. & 10 (45\%) & 125 & 12.50 & 30.50 & 3 (14\%) & 11 & 3.67 & 4.62 \\
\hline Other & 5 (42\%) & 14 & 2.80 & 4.02 & $3(25 \%)$ & 3 & 1.00 & - \\
\hline Total & $66(33 \%)$ & 336 & 5.09 & 12.38 & $32(16 \%)$ & 74 & 2.33 & 3.88 \\
\hline \multicolumn{9}{|l|}{$\begin{array}{l}\text { Dissociative } \\
\text { Identity Disorder }\end{array}$} \\
\hline Clin. Psych. & $10(9 \%)$ & 20 & 2.00 & 1.49 & $4(4 \%)$ & 4 & 1.00 & 0.82 \\
\hline Soc. W. & $3(5 \%)$ & 3 & 1.00 & - & 0 & - & - & - \\
\hline Psychiat. & $4(18 \%)$ & 7 & 1.75 & 0.96 & 3 (14\%) & 3 & 1.00 & - \\
\hline Other & 0 & - & - & - & 0 & - & - & - \\
\hline Total & $17(8 \%)$ & 30 & 1.76 & 1.25 & $7(3 \%)$ & 7 & 1.00 & 0.58 \\
\hline \multicolumn{9}{|c|}{$\begin{array}{l}\text { Dissociative Disorder-Not otherwise } \\
\text { specified (DDNOS) }\end{array}$} \\
\hline Clin. Psych. & 27 (25\%) & 58 & 2.15 & 1.61 & $9(8 \%)$ & 22 & 2.44 & 2.01 \\
\hline Soc. W. & $10(16 \%)$ & 29 & 2.90 & 2.64 & $5(8 \%)$ & 5 & 1.00 & - \\
\hline Psychiat. & $8(36 \%)$ & 65 & 8.13 & 16.97 & $3(25 \%)$ & 11 & 3.67 & 4.62 \\
\hline Other & 0 & - & - & - & 0 & - & - & - \\
\hline Total & 45 (22\%) & 152 & 3.38 & 7.13 & 17 (8\%) & 38 & 2.17 & 2.31 \\
\hline
\end{tabular}


in the caseloads of this Israeli sample. At the time the data were collected, $21 \%$ of the respondents were treating an amnesic client.

The next most frequently diagnosed DD was Depersonalization Disorder. One third of the sample had seen at least one patient with this problem, and $16 \%$ were treating the disorder at the time of the inquiry. Of the respondents, $22 \%$ had met at least one person with Dissociative Disorder Not Otherwise Specified (DDNOS), and in the practices of $8 \%$ of the clinicians surveyed, at least one DDNOS patient was currently being treated.

Eight percent of the sample had diagnosed a DID sometime during their careers and 3\% were treating DID when the data were collected. The rarest DD in Israel was Dissociative Fugue. Although 7\% of the sample had diagnosed at least one such case during their careers, only one person with this problem was being treated by the sampled clinicians when interviewed. The five most frequently considered alternative diagnoses to DID and the respective percentages of Israeli clinicians who reported on this clinical dilemma were: Borderline Personality Disorder (24\%), Psychotic Disorder/Schizophrenia (23\%), PTSD/Anxiety Disorder (10\%), Malingering (8\%), and Depressive Disorder (7\%). Respondents accounting for $17 \%$ of the sample said that they had disputed a DD/DID diagnosis made by another clinician at least once. In $44 \%$ of those instances the disagreeing respondents believed the erroneously diagnosed DID was actually suffering from a Borderline Personality Disorder. In $24 \%$ of them, respondents believed Psychotic Disorder/Schizophrenia was a more appropriate classification. Malingering should have replaced the erroneous DD/DID diagnosis in the view of $12 \%$ of the disputing clinicians.

Gender of therapist did not seem to be related to the tendency to diagnose any of the DDs. However, a tendency by male therapists to diagnose DID more than female therapists was identified (Chi-Square $=$ $2.78, \mathrm{DF}=1, \mathrm{p}=.09$ ). Inpatient clinicians did not rank DD/DID validity differently from outpatient clinicians. There was no difference between therapists working with youngsters and with adults concerning the validity they assigned to DD/DID or their likelihood to diagnose any of the DDs.

In their differential diagnoses of DDs, inpatient clinicians were more likely than outpatient clinicians to consider the option of Conduct Disorder (Chi-Square $=15.85, \mathrm{DF}=1, \mathrm{p}=.001$ ), Attention Deficit Hyperactivity Disorder $($ Chi-Square $=6.79, \mathrm{DF}=1, \mathrm{p}=.009)$, 
and Anxiety Disorder (Chi-Square $=6.51, \mathrm{DF}=1, \mathrm{p}=.01)$. Therapists working with children were more likely in their differential diagnoses to consider the possibilities of a sleep disorder (Chi Square $=4.76, \mathrm{DF}=1$, $\mathrm{p}=.03$ ) or Sexual Identity Disorder (Chi-square $=3.91, \mathrm{DF}=1, \mathrm{p}=.05$ ). Psychiatrists were more likely than other professionals to consider the alternative diagnosis of Malingering (Chi-Square $=11.47, \mathrm{DF}=3, \mathrm{p}=$ .009 ), Borderline Personality Disorder (Chi-Square $=10.93, \mathrm{DF}=3, \mathrm{p}=$ 0.12 ), Conduct Disorder (Chi-Square $=10.25, \mathrm{DF}=3, \mathrm{p}=0.17$ ), or Schizophrenia/Psychotic Disorder (Chi-Square $=9.15, \mathrm{DF}=3, \mathrm{p}=.027$ ).

There was a significant Spearman correlation between the degree of validity assigned to DDs and the total number of DDs diagnosed $(\mathrm{r}=$ $.28, \mathrm{p}=.002$ ). Interestingly, no relationship was found between the validity attributed to DID and the number of DIDs ever diagnosed $(r=\square .09, p=$ $.55)$.

The number of years of professional experience was not related to the validity assigned either to DDs or the DIDs, but the more experienced clinicians had been exposed to more sources of knowledge on $\mathrm{DD} / \mathrm{DID}(\mathrm{r}=.17, \mathrm{p}=.02)$, were less likely to show interest in continued education in the field $(\mathrm{r}=\square .17, \mathrm{p}=.03)$, and tended to have diagnosed more cases of DID $(\mathrm{r}=.47, \mathrm{p}=.059)$. When clinicians with less than 14 years of experience $(\mathrm{N}=25)$ were compared with those with 14 years of experience and more $(\mathrm{N}=43)$, the more experienced professionals had a higher number of career-long diagnoses of other DDs (Kruksal-Wallis Test Chi square $=6.28, \mathrm{DF}=1, \mathrm{p}=.01$ ).

Although $62.6 \%$ of the respondents reported never having encountered a DD, they had been exposed to a variety of information sources on the subject. Professional literature was a source of information on DD for $89.9 \%$ of the sample; $54.8 \%$ learned about DDs from a lecture they had attended, $38.5 \%$ from a documentary film, $37.2 \%$ from the media, and $32.2 \%$ acquired knowledge on this topic during professional supervision meetings (percentages do not add up to $100 \%$ because most respondents marked more than one source of knowledge). When asked if they would be interested in further education on DDs, $84.6 \%$ responded in the affirmative.

\section{DISCUSSION}

Skepticism regarding DDs is prevalent (e.g., Dell, 1988; Hacking, 1986, 1992; Kenny, 1986; Shorter, 1992; Spanos, 1989, 1994). A case 
in point is the ICD-10. This mental health classification method, which is widely used in Europe, assumes an agnostic posture as to the existence of DID "as something other than a culture-specific or even iatrogenic condition" (World Health Organization, 1993, p. 15).

This study aimed to enhance knowledge on familiarity with DDs, the belief in their validity, and the incidence of DDs in a culturally heterogeneous society in the Middle East.

In Israel, 211 mental health professionals were surveyed (about a $40 \%$ return rate). Results of this survey indicated that Israeli mental health professionals believe that dissociative disorders constitute viable diagnostic labels. To a lesser extent they also indicate a belief in the validity of DID. This outcome is comparable to two similar studies conducted in the United States (Cormier \& Thelen, 1998; Dunn et al., 1994; Hayes \& Mitchell, 1994), but is not in line with a more recent survey of American psychiatrists (Pope et al., 1999). That study limited its investigation to perceptions regarding dissociative amnesia and DID and reported that only about one-quarter of the respondents felt that these diagnoses were valid nosological entities. However, the authors did not control for familiarity with the disorders. In fact, familiarity may account for one of their findings-that psychodynamic psychiatrists were more likely than biological psychiatrists to support the validity of dissociative amnesia and DID.

Simpson (1989) suggested that DD/DID is an "iatrogenic culturebound not naturally occurring" phenomenon limited primarily to North America. However, the results of this study show that Israeli professionals are finding these concepts quite useful in their practice. The average Israeli mental health clinician has made 4.8 diagnoses of DDs during his or her career and carries at least one DD patient in his or her current caseload. At least one third of our respondents have had an opportunity to diagnose each of the following DDs: Dissociative Amnesia (42\%), DDNOS (36\%), and Depersonalization Disorder (33\%). Dissociative Amnesia appears to be a fairly common psychological problem in the caseloads of Israeli clinicians. Twenty-one percent of the respondents were treating an amnesic client when the data were collected. High prevalence of amnesia was previously reported among combat veterans [e.g., the literature on combat soldiers in World War II indicated a prevalence of amnesia ranging between $8.6 \%$ (Torrie, 1944) and 14.4\% (Sargent and Slater, 1941)], and among other traumatized populations [e.g., a prevalence of $59.6 \%$ among 
subjects with a reported history of childhood sexual abuse (Briere \& Conte, 1989)]. One possible explanation for the high incidence of amnesia among the caseloads of Israeli clinicians is related to the high exposure of Israelis to accumulated war related traumata. Many Israeli citizens were persecuted by the Nazis, survived at least one of the seven major Arab-Israeli wars, or were exposed to at least one of the countless other incidents of Middle East terror.

Another possible explanation for this phenomenon may be cultural. Okano (1997) reported that Japanese scholars have pointed out that dissociative fugue and dissociative amnesia appear to be more prevalent in Japan. Okano suggested that Japanese culture is often characterized as a "shame culture" that inhibits spontaneous expressions of negative emotions, thereby forcing some individuals to adopt dissociative solutions to their distress. Similar cultural factors may influence at least some Israelis. More than half of the Israeli population is comprised of Middle Eastern Jews and their offspring. Another 10\% are Arabs. The Middle Eastern culture is also described as a shame-oriented society rather than the more guilt-prone society the West appears to be (Somer \& Saadon, in press). Potential public shame and shunning act as an externalized super-ego and function as powerful controlling factors within this culture. The more externalized shame dynamics preclude any psychotherapeutic exploration of developmental processes, intrapsychic conflicts, or any meaningful confrontation of abusive authority figures within the family. From a Western perspective, traditional Middle Eastern societies are characterized by authoritarian male hierarchies often repressive to women and children. From early childhood members of traditional cultures in the Middle East are pressured to compromise their individuality and sacrifice it in exchange for the support and security provided by the family. As a result, many become detached from their true emotions and needs. Dwairy and Van Sickle (1996) claimed that repression is an inevitable consequence of traditional Middle Eastern society. As a result, the individual in this culture can become far removed from his or her self and tends to develop a false self that follows the practice of musayara (concealing one's true feelings and speaking and behaving in a manner that will be socially sanctioned) (Geriefat \& Katriel, 1989). The high incidence of dissociative amnesia reported in this study can also be understood as a result of the cultural difficulties of focusing on the 
self, communicating about it, and resolving intrapsychic conflicts that some Israeli patients may have experienced.

At the time of the inquiry, $3 \%$ of the respondents reported DSM-IVdefined cases of DID ( $8 \%$ had seen such cases at some point during their professional careers). These results are virtually identical to the $4 \%$ reported from Canada (Ross, Norton, \& Wozney, 1989) and to the $3 \%$ reported from Switzerland (Modestin, 1992).

Several factors could have biased these findings. One source of concern is the $39.5 \%$ response rate received in this study. Non-responders may have been less acquainted with dissociative symptomology, and may have seen patients with DD less frequently. It is also possible that a higher representation of dissociation skeptics existed among the non-responders than among those who chose to respond. However, the average return rates for several comparable surveys [Cormier \& Thelen, (1998); Dell, 1988; Dunn et al. (1994); Hayes \& Mitchell (1994); Mai (1995); McIntee (1998)] was 42.2\%. This figure is similar to the response rate achieved in this study.

Caution should also be used when interpreting the figures on reported past cases. Although respondents had a chance to check their records before they returned their questionnaires, some may have relied on memory when asked to report on their career-long encounters with DDs, and this might have resulted in some distortions. The topic of inquiry was fairly complex and may have compromised the reliability of the reported retrospective prevalence data. Nevertheless, the influence of memory bias is expected to be minimal or non-existent regarding data related to current caseloads and attitudes held at the time of inquiry.

Our findings indicate that the majority of Israeli mental health professionals do not hold extremely skeptical views about DDs/DID. Despite their geographical and cultural distance from North America, their attitudes are similar to those of colleagues in Canada (Mai, 1995) and the United States (Dunn et al., 1994). These data may strengthen the validity of this controversial clinical classification by showing that even in an ethnically heterogeneous Middle Eastern country the incidence of DDs/DID is very similar to that found in the Western hemisphere. However, Israeli respondents regarded DID as a less valid nosology than the other DDs, and more than $40 \%$ could not express an unequivocal "strong" or "complete" belief in the validity of DID. No other psychological phenomenon has sparked such heated debates 
concerning its scientific credibility. In fact, the only phenomenon that has attracted comparable scientific skepticism is parapsychology (Alcock \& Otis, 1980; Banziger, 1983; McClenon, 1982; McConnell \& Clark, 1991; Moss \& Butler, 1978; Palmer, 1986).

Several factors may explain the unique credibility problems that this DSM-IV and ICD-10 defined classification suffers, both in Israel and elsewhere:

\section{Divergence from Psychodynamic Psychology}

Toward the end of the 19th century, DID was considered a form of hysteria. In France the multiple personality diagnosis gained much of its prominence through the work of Janet (1907) who had also exerted a temporary influence on clinicians in the United States (e.g., Prince, 1908). During the same period, however, Freud developed his much more influential theories, which eventually superceded the incestual seduction hypothesis. The ensuing psychoanalytic movement abandoned Janet's notions of traumatic vertical dissociation of consciousness and developed the currently more popular models of object relations, ego- and self-psychology. Many Israeli clinicians are invested in current psychodynamic theory and technique, and would, therefore, have difficulty accommodating to the paradigm shift needed to adequately assess and treat DDs. When asked how traditional psychoanalysis conceptualizes and treats traumatogenic psychopathology, a leading Israeli analyst recently replied: "We don't do trauma."

\section{Positivist Skepticism and the Iatrogenesis Hypothesis}

Spanos (1996) posited that psychotherapy and therapy-related social supports can explain the genesis and maintenance of DID. Skeptics in Israel claim that some patients come to adopt a view of themselves that is congruent with the view conveyed to them by their therapists. Most of this incredulity is based on the belief that DIDs were iatrogenically created through suggestion and hypnosis (e.g., Mersky, 1992), a belief refuted in the literature (e.g., Steinberg, 1993; Ross \& Norton, 1989). During preparations for a staged trial on the validity of recovered memories of childhood sexual abuse held recently at the University of Haifa, heavy pressure was put on the organizers by positivist skeptics on campus to cancel the event. Their main argu- 
ment was that: "a respectable academic institution should not endorse controversial and scientifically dubious psychological concepts that can encourage dangerous clinical practice."

\section{The Malingering Claim}

Some Israeli clinicians have expressed their suspicions to me that DIDs are "a patient hoax aimed at secondary gains," such as the attraction of psychiatric attention, and the attaining of a desirable psychological diagnosis or the disowning of criminal responsibility (see also: Allison, 1981). This belief is held although (1) most DIDs were able to get psychiatric attention prior to their DID diagnosis, (2) treatment for DID is painful, demanding and lengthy, rather than a convenient "escape," and (3) most DIDs do not face criminal charges.

\section{Comorbidity of DID and DSM-IV Axis I and Axis II Disorders}

Presenting symptoms suggestive of depression were seen in $88 \%$ of the cases in an NIMH survey of DID (Putnam et al., 1986). Phobic, anxiety and panic, and post-traumatic disorders were often present in these patients (e.g., Coons, 1984). Psychotic and Schneiderian symptoms were also prevalent. The majority of DID patients experience auditory and/or visual hallucinations (e.g., Bliss, Larson, \& Nakashina, 1983), thought disorder (e.g., Putnam et al., 1984), and delusions (Putnam et al., 1986). Although our ability to differentiate DID from other Axis I disorders has become increasingly refined, many Israeli clinicians who are less familiar with DID symptomology may be expected to classify the more familiar comorbid signs as the building blocks of their diagnoses. To make matters even more complex, many DID patients present with an apparent mixed personality profile consisting of an array of avoidant, compulsive, borderline, narcissistic, and dependent features (Fink, 1991). Many of these clinical dilemmas were identified in the alternative diagnoses of the respondents in this study.

Associated with the incredulity problems in DID is the inevitably ensuing inaccuracy in diagnosing the disorder. Hayes and Mitchell (1994) showed that skepticism is directly related to inaccuracy in diagnosing DID, which can promote false negative errors. The author also found that skepticism and knowledge about DID were inversely 
related. It is hoped that the development of university curricula on DD, such as the course on the subject now offered by the author at the University of Haifa, will help improve knowledge on DD among future Israeli mental health professionals.

\section{REFERENCES}

Akyuz, G., Dogan, O., Sar, V., Yargic, L., \& Tutkun, H. (1996). Frequency of dissociative identity disorder in the general population: An epidemiologic study on a representative sample from a city in central Turkey. Program Book, 13th International Fall Conference, International Society for the Study of Dissociation. Glenview, IL: ISSD, p. 143.

Alcock, J.E. \& Otis, L.P. (1980). Critical thinking and belief in the paranormal. Psychological Reports, 46 (2), 479-482.

Aldridge-Morris, R. (1989). Multiple personality: An exercise in deception. Hillsdale, NJ: Lawrence Erlbaum.

Allison, R.B. (1981). Multiple personality and criminal behavior. American Journal of Forensic Psychiatry, 2, 32-38.

Altrocchi, J. (1992). "We don't have that problem here": MPD in New Zealand. Dissociation, 5 (2), 109-110.

American Psychiatric Association (1994). Diagnostic and Statistical Manual of Mental Disorders (4th ed.), Washington, DC: Author.

Banziger, G. (1983). Normalizing the paranormal: Short-term and long-term change in belief in paranormal among learners during a short course. Teaching of Psychology, 10 (4), 212-214.

Bliss, E.L. (1988). Commentary: Professional skepticism about multiple personality. Journal of Nervous and Mental Disease, 176 (9), 533-534.

Bliss, E.L., Larson, E.M. \& Nakashima, S.R. (1983). Auditory hallucinations and schizophrenia. Journal of Nervous and Mental Disease, 171, 30-33.

Bowers, K. (1991). Dissociation in hypnosis and multiple personality disorder. International Journal of Clinical and Experimental Hypnosis, 39 (3), 155-176.

Bowman, E.S. (1996, Nov. 8). A global update on the field of dissociative disorders. Presidential address of the scientific conference of the International Society for the Study of Dissociation. San Francisco, CA.

Braun, B.G. \& Sachs, R.G. (1985). The development of multiple personality disorder: Predisposing, precipitating and perpetuating factors. In R.P. Kluft (ed.), Childhood antecedents of multiple personality. Washington, DC: American Psychiatric Press, pp. 38-64.

Briere, J. \& Conte, J. (August, 1989). Amnesia in adults molested as children: Testing theories of repression. Paper presented at the annual meeting of the American Psychological Association, New Orleans, LA.

Carlson, E.B. \& Putnam, F.W. (1993). An update on the Dissociative Experiences Scale. Dissociation, 6, 16-27.

Clancy, G.P., Yates, W.R., \& Cadoret, R.J. (1993). Multiple Personality Disorder: Letter. Hospital and Community Psychiatry, 44 (3), 288. 
Coons, P.M. (1984). The differential diagnosis of multiple personality: A comprehensive review. Psychiatric Clinics of North America, 7, 51-65.

Cormier, J.F. \& Thelen, M.H. (1998). Professional skepticism of multiple personality disorder. Professional Psychology: Research and Practice, 29 (2), 163-167.

Davis, J.D. \& Davis, M.L. (1995). Prevalence of dissociative disorders in the mental health outpatient services of a British urban district. In O. van der Hart, S. Bown, \& N. Draijer (eds.), Proceedings of the Fifth Annual Spring Conference of the International Society for the Study of Dissociation. Amsterdam: ISSD, p. 78.

Davis, J.D. \& Davis, M.L. (1997). The prevalence of dissociative disorders within the mental health services of a British urban district. Paper presented at the Fourth Conference of the International Society for the Study of Dissociation. Chester, UK, April 19-11.

Dell, P.F. (1988). Professional skepticism about multiple personality. Journal of Nervous and Mental Disease, 176 (9), 528-531.

Dunn, G.E., Paolo, A.M., Ryan, J.J. \& van Fleet, J.N. (1994). Belief in the existence of multiple personality disorder among psychologists and psychiatrists. Journal of Clinical Psychology, 50 (3), 454-457.

Dwairy, M. \& Van Sickle, T.D. (1996). Western psychotherapy in traditional Arabic societies. Clinical Psychology Review, 16 (3), 231-249.

Fahy, T.A. (1988). The diagnosis of multiple personality disorder: A critical review. British Journal of Psychiatry, 153, 597-606.

Fink, D. (1991). The comorbidity of multiple personality disorder and DSM-III-R Axis II disorders. Psychiatric Clinics of North America, 14 (3), 547-566.

Frankel, F.H. (1990). Hypnotizability and dissociation. American Journal of Psychiatry, 147, 823-829.

Frischolz, E.J. \& Braun, B.G. (1990). “Is multiple personality disorder really rare in Japan?” Comment. Dissociation, 3 (2), 60-61.

Geriefat, Y. \& Katriel, T. (1989). Life demands Musayara: Communication and culture among Arabs in Israel. In S.T. Toomey \& F. Korzenny (Eds.), Language, communication and culture. London: Sage Publications.

Graves, S.M. (1989). Dissociative disorders and dissociative symptoms at a community mental health center. Dissociation, 2, 119-127.

Hacking, I. (1986). The invention of split personalities. In A. Donagan, A.N. Perovich, Jr., \& M.V. Wedin (eds.), Human nature and natural knowledge. New York: D. Reidel, pp. 63-85.

Hacking, I. (1992). Multiple personality and its hosts. History of the Human Sciences, 5, 3-31.

Hayes, J.A. \& Mitchell, J.C. (1994). Mental health professionals' skepticism about multiple personality disorder. Professional Psychology: Research and Practice, 25 (4), 410-415.

Horevitz, R. (1994). Dissociation and multiple personality: Conflicts and controversies. In S.J. Lynn \& J.W. Rhue (eds.), Dissociation: Clinical and Theoretical Perspectives. New York: The Guilford Press.

Janet, P. (1907). The major symptoms of hysteria: Fifteen lectures given in the medical school of Harvard University. New York: MacMillan. 
Kenny, M.G. (1986). The passion of Ansel Bourne: Multiple personality and American culture. Washington, DC: Smithsonian Institute Press.

Knudsen, H., Haslerud, J., Boe, T., Draijer, N., \& Boon, S. (1995). Prevalence of dissociative disorders in a Norwegian general psychiatric department (inpatients and daycare). In O. van der Hart, S. Boon, N. Dreijer, (eds). Proceedings of the Fifth Annual Spring Conference of the International Society for the Study of Dissociation, Amsterdam, Netherlands. Amsterdam: Bureau PAOG, p. 79.

Latz, T.T., Kramer, S.I., \& Hughes, D.L. (1995). Multiple personality disorder among female inpatients in a state hospital. American Journal of Psychiatry 152:1343-1348.

Mai, F.M. (1995). Psychiatrists' attitudes to multiple personality disorder: A questionnaire study. Canadian Journal of Psychiatry, 40, 154-157.

Margalit, H. \& Witzum, E. (1997a). Trauma, amnesia and dissociation: Clinical and theoretical aspects. Part I: A theoretical review. Sihot-Israel Journal of Psychotherapy, 11, 214-217 (in Hebrew).

Margalit, H. \& Witzum, E. (1997b). Trauma, amnesia and dissociation: Clinical and theoretical aspects. Part II: A case report. Sihot-Israel Journal of Psychotherapy, 12, 44-50 (in Hebrew).

Martinez-Taboas, A. (1990). Reflections on Takahashi's methodology and role of culture on MPD. Dissociation, 3 (2), 62-63.

Martinez-Taboas, A. (1991a). Multiple personality in Puerto Rico: Analysis of fifteen cases. Dissociation, 4, 189-192.

Martinez-Taboas, A. (1991b). Multiple personality as seen from a social constructionist viewpoint. Dissociation, 4, 129-133.

Martinez-Taboas, A. (1995a). Multiple personality: An Hispanic perspective. San Juan: PR: Paente Publications.

Martinez-Taboas, A. (1995b). The use of the Dissociative Experiences Scale in Puerto Rico. Dissociation, 8, 14-23.

McClenon, J. (1982). A survey of elite scientists: Their attitudes toward ESP and parapsychology. Journal of Parapsychology, 46 (2), 127-152.

McConnell, R.A. \& Clark, T.K. (1991). National Academy of Sciences' opinion on parapsychology. Journal of the American Society for Psychical Research, 85 (4), 333-365.

McIntee, J. (1998). Professional attitudes to Dissociative Identity Disorder (MPD) in Britain: More on treating DID where it doesn't exist. Paper presented at the Fourth Conference of the International Society for the Study of Dissociation. Chester, UK, April 1-3.

Merskey, H. (1992). The manufacture of personalities: The production of multiple personality disorder. British Journal of Psychiatry, 160, 327-340.

Merskey, H. (1993). Professional and lay opinions on multiple personality disorder. British Journal of Psychiatry, 162, 217.

Modestin, J. (1992) Multiple personality disorder in Switzerland. American Journal of Psychiatry, 149, 88-92.

Modestin, J., Ebner, G., Junghan, M., \& Erni, T. (1996). Dissociative experiences and dissociative disorders in acute psychiatric inpatients. Comprehensive Psychiatry 37:355-361. 
Moss, S. \& Butler, D.C. (1978). The scientific credibility of ESP. Perceptual and Motor Skills, 46 (3), 1063-1079.

Okano, K. (1997). A notion of “dissociogenic stress." Dissociation, 10 (2), 130-134.

Palmer, J. (1986). Professional skepticism: A critical approach to the psi controversy. Journal of Parapsychology, 50 (1), 29-42.

Piper, A. (1997). Hoax and reality: The bizarre world of multiple personality disorder. Northvale, NJ: Jason Aronson.

Pope, H.G., Oliva, P.S., Hudson, J.I., Bodkin, J.A. \& Gruber, A.J. (1999). Attitudes toward DSM-IV dissociative disorders diagnoses among board-certified American psychiatrists. American Journal of Psychiatry, 156 (2), 321-323.

Prince, M. (1908). The unconscious. Journal of Abnormal Psychology, 3, 261-297, 335-353, 391-426.

Putnam, F.W. (1989). Diagnosis and treatment of multiple personality disorder. New York: Guilford Press.

Putnam, F.W. (1996). Recent research on multiple personality disorder. Psychiatric Clinics of North America, 14, 489-517.

Putnam, F.W., Loewenstein, R.J., Silberman, E..\& Post, R.M. (1984). Multiple personality in a hospital setting. Journal of Clinical Psychiatry, 45, 172-175.

Putnam, F.W., Gurroff, J.J., Silberman, E.K., Barban, L. \& Post, R.M. (1986). The clinical phenomenology of multiple personality disorder: A review of 100 recent cases. Journal of Clinical Psychiatry, 47, 285-293.

Radwin, J.O. (1991). The multiple personality disorder: Has this trendy alibi lost its way? Law and Psychology Review, 16, 69-71.

Ross, C.A. (1990). "Is multiple personality disorder really rare in Japan?" Comment. Dissociation, 3 (2), 64-65.

Ross, C.A. (1991). Epidemiology of multiple personality disorder and dissociation. Psychiatric Clinics of North America, 14, 503-517.

Ross, C.A. \& Norton, G.R. (1989). Effects of hypnosis on the features of multiple personality disorder. American Journal of Clinical Hypnosis, 32 (2), 99-106.

Ross, C.A., Norton, G.R., \& Wozney, K. (1989). Multiple personality disorder: An analysis of 236 cases. Canadian Journal of Psychiatry, 34, 413-418.

ar, V., Yargic, C.I., \& Tutkun, H. (1996). Structured interview data on 35 cases of Dissociative Identity Disorder in Turkey. American Journal of Psychiatry, 153, 1329-1333.

ar, V., Tutkun, H., Alyanak, B., Bakim, B., \& Baral, I. (1996). Frequency of dissociative identity disorder among psychiatric outpatients in a Turkish university clinic. Program Book, 13th International Fall Conference, International Society for the Study of Dissociation, Glenview, IL: ISSD, p. 141.

Sargent, W. \& Slater, E. (1941). Amnestic syndromes in war. Proceeding of the Royal Society of Medicine, 34, 757-764.

Shorter, E. (1992). From paralysis to fatigue: A history of psychosomatic medicine in the modern era New York: Free Press.

Simpson, M.A. (1989). Multiple personality disorder (Letter). British Journal of Psychiatry, 155, 565.

Somer, E. (1987, November). Multiple personality: Diagnosis and treatment prin- 
ciples. Paper presented at the Third National Conference of the Israel Hypnosis Society. Tel Aviv (in Hebrew).

Somer, E. (1993). Possession syndrome in a histrionic personality: Exorcism and psychotherapy. Sihot-Israel Journal of Psychotherapy, 8, 40-47 (in Hebrew).

Somer, E. (1994). Delayed recall of child abuse: False memories or the next challenge in psychotherapy? Sihot-Israel Journal of Psychotherapy, 9, 46-50 (in Hebrew).

Somer, E. (1995). Trauma and trance and abreation in Dissociative Identity Disorder. Hypnoza, 1 (1), 8-15 (in Hebrew).

Somer, L. \& Somer, E. (1997). Psychodynamic perspectives on art work in Dissociative Identity Disorder. Sihot-Israel Journal of Psychotherapy, 11, 183-194 (in Hebrew).

Somer, E. \& Saadon, M. (in press). Stambali: Dissociative possession and trance in a Tunisian healing dance. Transcultural Psychiatry.

Spanos, N.P. (1989). Hypnosis, demonic possession and multiple personality: Strategic enactments and disavowal of responsibility for actions. In E.A. Word (ed.), Altered states of consciousness and mental health: Theoretical and methodological issues. Newbury Park, CA: Sage, pp. 96-124.

Spanos, N.P. (1994). Multiple identity enactments and multiple personality disorder: A sociocognitive perspective. Psychological Bulletin, 116 (1), 143-165.

Spanos, N.P. (1996). Multiple identities and false memories: A sociocognitive perspective. Washington, DC: American Psychological Association.

Steinberg, M. (1993). Structured clinical interview for DSM-IV dissociative disorders. Washington, DC: American Psychiatric Press.

Steinberg, M. (1993). Interviewer's guide to the structured clinical interview for DSM-IV dissociative disorders (SCID-D). Washington, DC: American Psychiatric Press.

Takahashi, Y. (1990). Is multiple personality disorder really rare in Japan? Dissociation, 3 (2), 57-59.

Thigpen, C.H. \& Cleckley, H.M. (1984). On the incidence of multiple personality: A brief communication. International Journal of Clinical and Experimental Hypnosis, 32, 63-66.

Torrie, A. (1944). Psychosomatic casualties in the Middle East. Lancet, 1, 139-143.

Tutkun, H., ar, V., Yargic, L., Ozpulat, T., Yanik, M., \& Kiziltan, E. (1996). Frequency of dissociative identity disorder among psychiatric inpatients in a Turkish university clinic. Program Book, 13th International Fall Conference International Society for the Study of Dissociation, Glenview, IL: ISSD, p. 139.

van der Hart, O. (1990). "Is multiple personality disorder really rare in Japan?" Comment. Dissociation, 3 (2), 66-67.

van der Hart, O. (1993). Multiple personality disorder in Europe: Impressions. Dissociation, 6 (2/3), 102-118.

Vanderlinden, J., Van Dyck, R., Vandereycken, W., \& Vertommen, H. (1991). Dissociated experiences in the general population in the Netherlands and Belgium: A study with the Dissociative Questionnaire (DIS-Q). Dissociation, 4, 180-184.

Vanderlinden, J., Van Dyck, R., Vandereycken, W., \& Vertommen, H. (1993). Dis- 
sociation and traumatic experiences in the general population of the Netherlands. Hospital \& Community Psychiatry, 44, 786-788.

World Health Organization (1993). The ICD-10 classification of mental and behavioral disorders: Diagnostic criteria for research. Geneva: World Health Organization.

SUBMITTED: 3/06/99

REVISION SUBMITTED: 5/27/99

ACCEPTED: 5/28/99

for faculty/professionals with journal subscription recommendation authority for their institutional library...

If you have read a reprint or photocopy of this article, would you like to make sure that your library also subscribes to this journal? If you have the authority to recommend subscriptions to your library, we will send you a free sample copy for review with your librarian. Just fill out the form below-and make sure that you type or write out clearly both the name of the journal and your own name and address.

( ) Yes, please send me a complimentary sample copy of this journal:

(please write in complete journal title here-do not leave blank)

I will show this journal to our institutional or agency library for a possible subscription.

The name of my institutional/agency library is:

NAME:

INSTITUTION:

ADDRESS:

CITY: STATE: ZIP:

Return to: Sample Copy Department, The Haworth Press, Inc., 10 Alice Street, Binghamton, NY 13904-1580 